Research Article

\title{
On the Convergence of Solutions for SPDEs under Perturbation of the Domain
}

\author{
Zhongkai Guo, ${ }^{1}$ Jicheng Liu, ${ }^{2}$ and Wenya Wang ${ }^{2,3}$ \\ ${ }^{1}$ School of Mathematics and Statistics, South-Central University for Nationalities, Wuhan 430074, China \\ ${ }^{2}$ School of Mathematics and Statistics, Huazhong University of Science and Technology, Wuhan 430074, China \\ ${ }^{3}$ School of Mathematics and Computer Science, Jianghan University, Wuhan 430056, China
}

Correspondence should be addressed to Wenya Wang; wangwenya2014@gmail.com

Received 26 January 2016; Accepted 30 May 2016

Academic Editor: Nikos I. Karachalios

Copyright @ 2016 Zhongkai Guo et al. This is an open access article distributed under the Creative Commons Attribution License, which permits unrestricted use, distribution, and reproduction in any medium, provided the original work is properly cited.

We investigate the effect of domain perturbation on the behavior of mild solutions for a class of semilinear stochastic partial differential equations subject to the Dirichlet boundary condition. Under some assumptions, we obtain an estimate for the mild solutions under changes of the domain.

\section{Introduction}

Domain perturbation, or sometimes referred to as "perturbation of the boundary," for boundary value problems is a special topic in perturbation problems. The motivation to study domain perturbation comes from various sources, which include shape optimization, solution structure of nonlinear problems, and numerical analysis. The main characteristic of domain perturbation is that the operators and the nonlinear terms live in different spaces, which leads to the solutions of partial differential equations living in different spaces. The fundamental question of domain perturbation is to consider how solutions behave upon varying domains. However, when we only consider the case of smooth perturbation of the domain, we could perform a change of variables to convert the perturbation problem into a fixed domain problem which is only perturbation of the coefficients. In this case, domain perturbation becomes back to a standard perturbation problem; in turn we may apply standard techniques such as the implicit function theorem, the Lyapunov-Schmidt method, and the transversality theorem to study it. Nevertheless, difficulties arrive when the change of variables and other standard tools do not work (see [1]) such as singular perturbation. So, it is more challenging and interesting to consider singular perturbation problems.
There are lots of papers concerning this topic [2-10]. For elliptic equations, see $[2,3]$ and references therein. In [3], the authors give a sufficient condition on domains which guarantees the spectrum behaviors continuously. It is very clear that the spectral behavior of linear operators is extremely important when analyzing the continuity properties for domain perturbation problem. The work of [2] obtains the convergence of solution for elliptic equation subject to Dirichlet boundary condition; necessary and sufficient conditions are discussed for strong and uniform convergence for the corresponding resolvent operators. In [6] the author gets the persistence of periodic solutions and convergence of solutions for both linear and semilinear parabolic initial value problems subject to Dirichlet boundary condition, and [10] does so for evolution equation. In [7, 8] authors study the convergence of invariant manifolds under the perturbation of the domain. For stochastic system, we recommend [9], caring about the coefficients perturbation for semilinear stochastic partial differential equations; as we mention above it belongs to smooth domain perturbation problem. We do not attempt here to give a complete bibliography but make a rather arbitrary choice of references.

Notice that all of works as we mentioned above are under the condition of Mosco convergence which describes the domain perturbation. For Dirichlet problems, it is worth 
pointing out that the condition of Mosco convergence conditions is equivalent to the strong convergence of resolvent operators (see [6, Theorem 5.2.4]), which is weaker than the operator norm convergence of resolvent operators.

Under the condition of the operator norm convergence of resolvent operators, the author of [11] gives a distance estimate of the inertial manifolds for partial differential equations of evolutionary type under perturbation of the domain. As there are not many results on domain perturbation with noise in the dynamics, inspired by [11], we take similar conditions as in [11] to consider the convergence of solution for stochastic partial differential equations under perturbation of the domain. We show how the mild solution of the stochastic differential equations behaves when domain $\Omega_{\epsilon}$ converges to domain $\Omega$ under a certain sense.

This paper is organized as follows: In Section 2, we review the results of the existence and uniqueness to the stochastic partial differential equation which we consider. In Section 3, we use the relationship between the resolvent operator and the semigroup to deduce the convergence of the semigroup on $[r, T]$. To get the result of convergence of solutions, we divide the interval $[0, T]$ into two parts as $[0, r]$ and $[r, T]$; here $r>0$ and is sufficiently small. Then we take estimate, respectively, for each part to get our results.

Throughout this paper, the letter $C$ below will denote positive constants whose value may change in different occasions. We will write the dependence of constant on parameters explicitly if it is essential.

\section{Preliminaries}

Let $H$ be an infinite dimensional separable Hilbert space with norm $\|\cdot\|$. Let the sectorial operator $A: D(A) \rightarrow H$ be a self-adjoint positive linear operator with a compact resolvent. Then the spectrum of $A$ is real. We denote its spectrum by

$$
\sigma(A)=\left\{\lambda_{n}\right\}_{n=1}^{\infty}, \quad 0<c \leq \lambda_{1} \leq \lambda_{2} \leq \cdots \leq \lambda_{n} \leq \cdots,
$$

and an associated orthonormal family of eigenfunctions by $\left\{\phi_{n}\right\}_{n=1}^{\infty}$. Since $A$ is a self-adjoint sectorial operator, $-A$ is the infinitesimal generator of an analytic semigroup, which is denoted by

$$
e^{-A t}=\frac{1}{2 \pi i} \int_{\gamma}(\lambda I+A)^{-1} e^{\lambda t} d \lambda
$$

where $\gamma$ is a contour in the resolvent set of $-A$. Also, since $A$ is a self-adjoint sectorial operator, the representation of $e^{-A t}$ above is equivalent to

$$
e^{-A t} u=\sum_{n=1}^{\infty} e^{-\lambda_{n} t}\left(u, \phi_{n}\right) \phi_{n} .
$$

By the definition $e^{-A t}$, we can easily get the following estimate:

$$
\left\|e^{-A t}\right\|_{L(H, H)} \leq e^{-\lambda_{1} t} \leq 1,
$$

for $t \geq 0$, which implies that $e^{-A t}$ is an analytic contraction semigroup.
We consider the stochastic equation as follows:

$$
\begin{aligned}
d u(t)+A u(t) d t & =f(t, u(t)) d t+g(t, u(t)) d w(t), \\
t \in(0, T], & \\
u(0) & =u_{0} \in H .
\end{aligned}
$$

Here $A$ is a sectorial operator; $w(t)$ is a scalar Wiener process on a probability space $(\Omega, \mathscr{F}, \mathbb{P})$. In addition, suppose that, for a.e. $(t, \omega) \in[0, T] \times \Omega=: \Omega_{T}$, the drift coefficients $f(t, \cdot, \omega): \Omega_{T} \times H \rightarrow H$ and diffusion coefficients $g(t, \cdot, \omega)$ : $\Omega_{T} \times H \rightarrow H$ are $\mathscr{F}_{t}$-adapted and satisfy certain conditions.

We adopt the following assumptions throughout this paper.

(A.1) There exists a constant $k>0$ such that, for any $u(t), v(t) \in H$, the Lipschitz continuity condition holds:

$$
\begin{aligned}
\|f(t, \omega, u(t))-f(t, \omega, v(t))\|_{H}^{2} \\
\quad+\|g(t, \omega, u(t))-g(t, \omega, v(t))\|_{H}^{2} \\
\leq k\|u(t)-v(t)\|_{H}^{2} ;
\end{aligned}
$$

here $(t, \omega) \in \Omega_{T}$ and $u(t), v(t) \in H$.

Notice that (A.1) implies there exists a constant $C$ such that

$$
\begin{aligned}
& \|f(t, \omega, u(t))\|_{H}^{2}+\|g(t, \omega, u(t))\|_{H}^{2} \\
& \quad \leq C\left(1+\|u(t)\|_{H}^{2}\right), \quad(t, \omega) \in \Omega_{T},
\end{aligned}
$$

for any $u(t) \in H$.

Now we introduce the definition of mild solution to (5). Taking the classic method for proving the existence and uniqueness of solution as $[12,13]$, we can deduce the existence and uniqueness of solution for (5), which is represented as follows.

Definition 1 (mild solution). An $H$-valued predictable process $u(t)$ is called a mild solution of (5) if for any $t \in[0, T]$

$$
\begin{aligned}
u(t)= & e^{-A t} u_{0}+\int_{0}^{t} e^{-A(t-s)} f(s, u(s)) d s \\
& +\int_{0}^{t} e^{-A(t-s)} g(s, u(s)) d w(s) .
\end{aligned}
$$

Let $X_{T}$ denote the set of all continuous $\mathscr{F}_{t}$-adapted processes valued in $H$ for $0 \leq t \leq T$ such that $E \sup _{0 \leq t \leq T}\|u(t)\|^{2}<$ $\infty$. Then $X_{T}$ is a Banach space under the norm

$$
\|u\|_{T}=E \sup _{0 \leq t \leq T}\|u(t)\|^{2} .
$$

Define an operator $\Gamma$ in $X_{T}$ as follows:

$$
\begin{aligned}
\Gamma u(t)= & e^{-A t} u_{0}+\int_{0}^{t} e^{-A(t-s)} f(s, u(s)) d s \\
& +\int_{0}^{t} e^{-A(t-s)} g(s, u(s)) d w(s)
\end{aligned}
$$


for $u \in X_{T}$. It is easy to prove that the operator $\Gamma$ is well defined and Lipschitz continuous in $X_{T}$. Then by the contraction mapping principle, it is easy to prove the existence and uniqueness of mild solution for (5) as the following.

Theorem 2 (existence and uniqueness). Suppose the condition (A.1) holds true, and let $u_{0}$ be $\mathscr{F}_{0}$-measurable random field such that $E\left\|u_{0}\right\|^{2}<\infty$. Then the initial-boundary value problem for (5) has a unique mild solution $u(t)$ which is a continuous adapted process in $H$ such that $u \in L^{2}(\Omega ; C([0, T] ; H))$ and

$$
E \sup _{0 \leq t \leq T}\|u(t)\|^{2} \leq C\left(1+E\left\|u_{0}\right\|^{2}\right)
$$

for some constant $C>0$.

\section{Solution under Perturbation of the Domain}

In this section, we consider the following perturbation problem of (5):

$$
\begin{aligned}
d u^{\epsilon}(t)+A_{\epsilon} u^{\epsilon}(t) d t= & f^{\epsilon}\left(t, u^{\epsilon}(t)\right) d t \\
& +g^{\epsilon}\left(t, u^{\epsilon}(t)\right) d w(t), \\
& t \in[0, T], \\
u^{\epsilon}(0)= & u_{0}^{\epsilon},
\end{aligned}
$$

for $\epsilon>0$, where $A_{\epsilon}: D\left(A_{\epsilon}\right) \subset H^{\epsilon} \rightarrow H^{\epsilon}$ is a selfadjoint positive linear operator on a Hilbert space $H^{\epsilon}$ with norm $\|\cdot\|_{\epsilon}$, and let $u_{0}^{\epsilon}$ be $\mathscr{F}_{0}$-measurable random field such that $E\left\|u_{0}^{\epsilon}\right\|^{2}<\infty$. We also assume that the nonlinear terms $f^{\epsilon}(t, \omega, u(t)): \Omega_{T} \times H^{\epsilon} \rightarrow H^{\epsilon}$ and $g^{\epsilon}(t, \omega, u(t)): \Omega_{T} \times H^{\epsilon} \rightarrow$ $H^{\epsilon}$ satisfy (A.1), which guarantees the existence and unique of mild solution to (12). By Theorem 2, for each $\epsilon>0$, there is an $H$-valued continuous $\mathscr{F}_{t}$-adapted process $u^{\epsilon}(t)$ such that

$$
\begin{aligned}
u^{\epsilon}(t)= & e^{-A_{\epsilon} t} u_{0}^{\epsilon}+\int_{0}^{t} e^{-A_{\epsilon}(t-s)} f\left(s, u^{\epsilon}(s)\right) d s \\
& +\int_{0}^{t} e^{-A_{\epsilon}(t-s)} g\left(s, u^{\epsilon}(s)\right) d w(s)
\end{aligned}
$$

for any $t \in[0, T]$.

Note the solutions value in different function spaces $H^{\epsilon}$ for different $\epsilon$. To deal with domain perturbation, we assume there exist bound linear operators $\mathbf{P}$ and $\mathbf{Q}$ such that

$$
\begin{aligned}
& \mathbf{P}: H \longrightarrow H^{\epsilon}, \\
& \mathbf{Q}: H^{\epsilon} \longrightarrow H, \\
& \mathbf{Q} \circ \mathbf{P}=I, \\
&\|\mathbf{P}\|_{\mathscr{L}\left(H, H^{\epsilon}\right)} \leq C, \\
&\|\mathbf{Q}\|_{\mathscr{L}\left(H^{\epsilon}, H\right)} \leq C, \\
&\|\mathbf{P} u(t)\|_{H^{\epsilon}}
\end{aligned}
$$

To derive the solution of (12) converging to the solution of (5), we also impose the following hypotheses:

(H.1) For $A$ and $A_{\epsilon}$, we assume

$$
\left\|A_{\epsilon}^{-1} \mathbf{P}-\mathbf{P} A^{-1}\right\|_{\mathscr{L}\left(H, H^{\epsilon}\right)}=\tau(\epsilon) \longrightarrow 0 \quad \text { as } \epsilon \longrightarrow 0 .
$$

(H.2) We assume that the nonlinear terms $g^{\epsilon}, f^{\epsilon}: \Omega_{T} \times$ $H^{\epsilon} \rightarrow H^{\epsilon}$ for $0 \leq \epsilon \leq \epsilon_{0}$ satisfy the following:

(i) $f^{\epsilon}$ and $g^{\epsilon}$ approximate $f$ and $g$ in the following sense:

$$
\sup _{0 \leq t \leq T}\left\|f^{\epsilon}(\mathbf{P} u(t))-\mathbf{P} f(u(t))\right\|_{H^{e}}^{2}=\tau_{1}(\epsilon) \longrightarrow 0,
$$$$
\text { as } \epsilon \longrightarrow 0 \text {, }
$$

$$
\sup _{0 \leq t \leq T}\left\|g^{\epsilon}(\mathbf{P} u(t))-\mathbf{P} g(u(t))\right\|_{H^{\epsilon}}^{2}=\tau_{1}(\epsilon) \longrightarrow 0,
$$$$
\text { as } \epsilon \longrightarrow 0 \text {. }
$$

(ii) $f, g$ and $f^{\epsilon}, g^{\epsilon}$ have the uniformly bounded support and satisfy the following estimate:

$$
E \sup _{0 \leq t \leq T}\|g(u(t))\|^{2} \leq C(T, R),
$$

same assumption also for $f, f^{\epsilon}, g^{\epsilon}$. Here $C(T, R)$ denotes a constant related to $T, R$ and $R$ denotes the radius of the support.

(H.3) For initial value of $u_{0}$ and $u_{0}^{\epsilon}$, we assume $u_{0} \in D(A)$, $u_{0}^{\epsilon} \in D\left(A^{\epsilon}\right)$, and $E\left\|u_{0}^{\epsilon}-\mathbf{P} u_{0}\right\|_{H^{\epsilon}}^{2}=\tau_{0}(\epsilon) \rightarrow 0$, as $\epsilon \rightarrow 0$.

By hypothesis (H.1) we have the following result, which concerns the relationship of spectrum between $A$ and $A_{\epsilon}$ (see [11]).

Lemma 3 (upper semicontinuity of spectrum). If $K_{0}$ is a compact set of the complex plane with $K_{0} \subset \rho(-A)$, the resolvent set of $A$, and hypothesis (H.1) is satisfied, then there exists $\epsilon_{0}\left(K_{0}\right)>$ such that $K_{0} \subset \rho\left(-A_{\epsilon}\right)$ for all $0<\epsilon \leq \epsilon_{0}\left(K_{0}\right)$. Moreover, one has the estimates

$$
\left\|\left(\lambda I-A_{\epsilon}\right)^{-1}\right\|_{\mathscr{L}\left(H^{\epsilon}, H^{\epsilon}\right)} \leq C\left(K_{0}\right)
$$

for all $\lambda \in K_{0}, 0<\epsilon \leq \epsilon_{0}\left(K_{0}\right)$.

The result implies the upper semicontinuity of the spectrum; that is, if $\lambda_{\epsilon} \in \sigma\left(A_{\epsilon}\right)$ and $\lambda^{\epsilon} \rightarrow \lambda$ then $\lambda \in \sigma(A)$. Also we have the resolvent operator estimate as the following (see [11]).

Lemma 4 (operator norm convergence of resolvent operators). Let the condition (H.1) be satisfied; if $\lambda \in \rho(-A)$ and $\epsilon$ is small enough so that $\lambda \in \rho\left(-A_{\epsilon}\right)$, one has

$$
\begin{aligned}
& \left\|\left(\lambda+A_{\epsilon}\right)^{-1} \mathbf{P}-\mathbf{P}(\lambda+A)^{-1}\right\|_{\mathscr{L}\left(H, H^{\epsilon}\right)} \leq C(\epsilon, \lambda) \tau(\epsilon) \\
& \quad \longrightarrow 0, \quad \text { as } \epsilon \longrightarrow 0 .
\end{aligned}
$$


By the relationship of spectrum and resolvent set, we have that hypothesis (H.1) is equivalent to the operator norm convergence of resolvent operators. To compare condition (H.1) with Mosco convergence condition, we quote the Mosco convergence condition as the following.

Let $V$ be a reflexive and separable Banach space and let $K_{n}, K$ be closed and convex subsets of $V$. We say that $K_{n}$ converges to $K$ in the sense of Mosco if the following conditions hold (see [14]):

(1) For every $u \in K$, there exists a sequence $u_{n} \in K_{n}$ such that $u_{n} \rightarrow u$ in $V$ strongly.

(2) If $\left(n_{k}\right)$ is a sequence of indices converging to $\infty,\left(u_{k}\right)$ is a sequence such that $u_{k} \in K_{n_{k}}$ for every $k$ and $u_{k} \rightarrow u$ in $V$ weakly; then $u \in K$.

As we mentioned in Introduction, hypothesis (H.1) is stronger than the Mosco condition, which is equivalent to the strong convergence of resolvent operators for Dirichlet Problem. For details about this notation see Daners [6].

As we all know, the relationship between resolvent operator and semigroup is denoted by

$$
e^{-A t}=\frac{1}{2 \pi i} \int_{\gamma}(\lambda I+A)^{-1} e^{\lambda t} d \lambda,
$$

where $\gamma$ is the boundary of $\Sigma_{-a, \phi}=\{\lambda \in \mathbb{C}:|\arg (\lambda+a)| \leq$ $\pi-\phi\} \subset \rho(-A), \phi \in(0, \pi / 2)$. Simply we take $a=0, \phi=\pi / 4$. Then we have

$$
\begin{aligned}
\gamma & =\gamma_{1} \cup \gamma_{2} \\
& =\left\{\delta e^{-i(3 \pi / 4)}: 0 \leq \delta<\infty\right\} \cup\left\{r e^{i(3 \pi / 4)}: 0 \leq \delta<\infty\right\}
\end{aligned}
$$

and $C(\epsilon, \lambda) \leq 6$ for all $\lambda \in \Sigma_{0, \pi / 4}$. From Lemma 4 we have the following estimate.

Lemma 5 (convergence of semigroup). Let (H.1) be satisfied. Then one has

$$
\left\|e^{-A_{\epsilon} t} \mathbf{P}-\mathbf{P} e^{-A t}\right\|_{\mathscr{L}\left(H, H^{e}\right)} \leq \frac{C}{r} \tau(\epsilon) \longrightarrow 0,
$$

$$
\text { as } \epsilon \longrightarrow 0 \text {, }
$$

for any $t \in[r, T]$; here $r>0$.

Proof. By (20) and Lemma 4, we can estimate

$$
\begin{aligned}
& \left\|e^{-A_{\epsilon} t} \mathbf{P}-\mathbf{P} e^{-A t}\right\|_{\mathscr{L}\left(H, H^{e}\right)} \\
& =\| \frac{1}{2 \pi i} \int_{\gamma}\left(\lambda I+A_{\epsilon}\right)^{-1} \mathbf{P} e^{\lambda t} d \lambda \\
& \quad-\frac{1}{2 \pi i} \int_{\gamma} \mathbf{P}(\lambda I+A)^{-1} e^{\lambda t} d \lambda \|_{\mathscr{L}\left(H, H^{\epsilon}\right)} \\
& \quad \leq C\left|\int_{\gamma_{1} \cup \gamma_{2}} \tau(\epsilon) e^{\lambda t} d \lambda\right| .
\end{aligned}
$$

For $\lambda \in \gamma_{1} \cup \gamma_{2}$, we compute $\left|e^{\lambda t}\right|=\left|e^{\delta t e^{-i(3 \pi / 4)}}\right|=e^{-(\sqrt{2} / 2) \delta t}$ with $0 \leq \delta<+\infty$. Then we have

$$
\begin{aligned}
\left\|e^{-A_{\epsilon} t} \mathbf{P}-\mathbf{P} e^{-A t}\right\|_{\mathscr{L}\left(H, H^{\epsilon}\right)} \leq C \tau(\epsilon) \int_{0}^{+\infty} e^{-(\sqrt{2} / 2) \delta t} d r \\
\quad \leq \frac{C}{t} \tau(\epsilon) .
\end{aligned}
$$

Hence by (H.2) we get

$$
\begin{array}{ll}
\left\|e^{-A_{\epsilon} t} \mathbf{P}-\mathbf{P} e^{-A t}\right\|_{\mathscr{L}\left(H, H^{\epsilon}\right)} \leq \frac{C}{r} \tau(\epsilon) \longrightarrow 0, & \\
& \text { as } \epsilon \longrightarrow 0,
\end{array}
$$

for any $t \in[r, T]$; here $r>0$.

Lemma 6. Let $A$ be a sectorial operator; if $X \in D(A)$, then one has the following estimate:

$$
\left\|\left(e^{-A r}-I\right) X\right\| \leq C r\|A X\| .
$$

Proof. For $r>\tau>0$ we have

$$
\begin{aligned}
\left(e^{-A r}-e^{-A \tau}\right) X & =-A \int_{\tau}^{r} e^{-A s} X d s \\
& =-\int_{\tau}^{r} A e^{-A s} X d s, \quad \forall X \in D(A) .
\end{aligned}
$$

Because $A$ is a sectorial operator, we have $A e^{-A r} X=e^{-A r} A X$ and then

$$
\begin{aligned}
\int_{\tau}^{r}\left\|A e^{-A s} X\right\| d s & =\int_{\mathcal{\tau}}^{r}\left\|e^{-A s} A X\right\| d s \leq C \int_{\tau}^{r}\|A X\| d s \\
& \leq C r\|A X\| .
\end{aligned}
$$

Let $\tau \rightarrow 0^{+}$; then we get our result.

Now we state and prove our main result as the following.

Theorem 7 (convergence of the solutions). Suppose (H.1) to (H.3) and (A.1) hold true. Then one has

$$
\begin{gathered}
E \sup _{0 \leq t \leq T}\left\|u^{\epsilon}(t)-\mathbf{P} u(t)\right\|_{H^{\epsilon}}^{2} \leq e^{C(T) k^{2}}[C(T, R) \\
\left.\cdot\left(r^{2}+r+\tau_{0}(\epsilon)+\tau_{1}(\epsilon)+\frac{\tau(\epsilon)^{2}}{r^{2}}+\frac{\tau(\epsilon)}{r}\right)\right] .
\end{gathered}
$$

In particular,

$$
E \sup _{0 \leq t \leq T}\left\|u^{\epsilon}(t)-\mathbf{P} u(t)\right\|_{H^{\epsilon}}^{2} \longrightarrow 0,
$$

when we first let $\epsilon \rightarrow 0$ and then $r \rightarrow 0$. 
Proof. From (8) and (13), we have

$$
\begin{aligned}
E \sup _{0 \leq t \leq T} & \left\|u^{\epsilon}(t)-\mathbf{P} u(t)\right\|_{H^{\epsilon}}^{2}=E \sup _{0 \leq t \leq T} \| e^{-A_{\epsilon} t} u_{0}^{\epsilon}-\mathbf{P} e^{-A t} u_{0} \\
& +\int_{0}^{t} e^{-A_{\epsilon}(t-s)} f^{\epsilon}\left(s, u^{\epsilon}(s)\right)-\mathbf{P} e^{-A(t-s)} f(s, u(s)) d s \\
& +\int_{0}^{t} e^{-A_{\epsilon}(t-s)} g^{\epsilon}\left(s, u^{\epsilon}(s)\right) \\
& -\mathbf{P} e^{-A(t-s)} g(s, u(s)) d w(s) \|_{H^{\epsilon}}^{2} \leq 3 E \\
& \cdot \sup _{0 \leq t \leq T}\left\|e^{-A_{\epsilon} t} u_{0}^{\epsilon}-\mathbf{P} e^{-A t} u_{0}\right\|_{H^{\epsilon}}^{2}+3 E \\
& \cdot \sup _{0 \leq t \leq T} \| \int_{0}^{t} e^{-A_{\epsilon}(t-s)} f^{\epsilon}\left(s, u^{\epsilon}(s)\right) \\
& -\mathbf{P} e^{-A(t-s)} f(s, u(s)) d s \|_{H^{\epsilon}}^{2}+3 E \\
+ & 3 I_{3} . \\
& +\sup _{0 \leq t \leq T} \| \int_{0}^{t} e^{-A_{\epsilon}(t-s)} g^{\epsilon}\left(s, u^{\epsilon}(s)\right) \\
& \mathbf{P} e^{-A(t-s)} g(s, u(s)) d w(s) \|_{H^{\epsilon}}^{2}=: 3 I_{1}+3 I_{2} \\
&
\end{aligned}
$$

Next we will estimate $I_{1}, I_{2}$, and $I_{3}$, respectively. Fix $r$ sufficient small. For $I_{1}$ we have

$$
\begin{aligned}
I_{1} \leq & E \sup _{r \leq t \leq T}\left\|e^{-A_{\epsilon} t} u_{0}^{\epsilon}-\mathbf{P} e^{-A t} u_{0}\right\|_{H^{\epsilon}}^{2} \\
& +\underset{0 \leq t \leq r}{E \sup _{0}}\left\|e^{-A_{\epsilon} t} u_{0}^{\epsilon}-\mathbf{P} e^{-A t} u_{0}\right\|_{H^{\epsilon}}^{2}
\end{aligned}
$$

with

$$
\begin{aligned}
& E \sup _{r \leq t \leq T}\left\|e^{-A_{\epsilon} t} u_{0}^{\epsilon}-\mathbf{P} e^{-A t} u_{0}\right\|_{H^{\epsilon}}^{2} \\
& \leq 2 E \sup _{r \leq t \leq T}\left\|e^{-A_{\epsilon} t} u_{0}^{\epsilon}-e^{-A_{\epsilon} t} \mathbf{P} u_{0}\right\|_{H^{\epsilon}}^{2} \\
& +2 E \sup _{r \leq t \leq T}\left\|e^{-A_{\epsilon} t} \mathbf{P} u_{0}-\mathbf{P} e^{-A t} u_{0}\right\|_{H^{\epsilon}}^{2} \\
& \leq 2 E \sup _{r \leq t \leq T}\left\|u_{0}^{\epsilon}-\mathbf{P} u_{0}\right\|_{H^{e}}^{2}+C \frac{\tau(\epsilon)}{r} \\
& =C\left(\frac{\tau(\epsilon)}{r}+\tau_{0}(\epsilon)\right) \text {, } \\
& E \sup _{0 \leq t \leq r}\left\|e^{-A_{\epsilon} t} u_{0}^{\epsilon}-\mathbf{P} e^{-A t} u_{0}\right\|_{H^{\epsilon}}^{2} \\
& \leq 3 E \sup _{0 \leq t \leq r}\left\|e^{-A_{\epsilon} t} u_{0}^{\epsilon}-u_{0}^{\epsilon}\right\|_{H^{\epsilon}}^{2}
\end{aligned}
$$

$$
\begin{aligned}
& +3 E \sup _{0 \leq t \leq r}\left\|\mathbf{P} u_{0}-\mathbf{P} e^{-A t} u_{0}\right\|_{H^{\epsilon}}^{2} \\
& +3 E \sup _{0 \leq t \leq r}\left\|u_{0}^{\epsilon}-\mathbf{P} u_{0}\right\|_{H^{\epsilon}}^{2} \leq C\left(\tau_{0}(\epsilon)+r\right) .
\end{aligned}
$$

Here the contraction property of $e^{-A t}$, Lemma 6, and (H.3) are used. Then we obtain

$$
I_{1} \leq C\left(\frac{\tau(\epsilon)}{r}+\tau_{0}(\epsilon)+r\right) .
$$

For $I_{2}$ we have

$$
\begin{aligned}
I_{2} & \leq 2 E \sup _{0 \leq t \leq T}\left\|\int_{0}^{t} e^{-A_{\epsilon}(t-s)}\left(f^{\epsilon}\left(s, u^{\epsilon}(s)\right)-\mathbf{P} f(s, u(s))\right)\right\|_{H^{\epsilon}}^{2} \\
& +2 E \sup _{0 \leq t \leq T}\left\|\int_{0}^{t}\left(e^{-A_{\epsilon}(t-s)} \mathbf{P}-\mathbf{P} e^{-A(t-s)}\right) f(s, u(s)) d s\right\|_{H^{\epsilon}}^{2} \\
& \leq 4 E \\
& \cdot \sup _{0 \leq t \leq T}\left\|\int_{0}^{t} e^{-A_{\epsilon}(t-s)}\left(f^{\epsilon}\left(s, u^{\epsilon}(s)\right)-f^{\epsilon}(s, \mathbf{P} u(s))\right) d s\right\|_{H^{\epsilon}}^{2} \\
& +4 E \\
& \cdot \sup _{0 \leq t \leq T}\left\|\int_{0}^{t} e^{-A_{\epsilon}(t-s)}\left(f^{\epsilon}(s, \mathbf{P} u(s))-\mathbf{P} f(s, u(s))\right) d s\right\|_{H^{\epsilon}}^{2} \\
& +2 E \sup _{0 \leq t \leq T}\left\|\int_{0}^{t}\left(e^{-A_{\epsilon}(t-s)} \mathbf{P}-\mathbf{P} e^{-A(t-s)}\right) f(s, u(s)) d s\right\|_{H^{\epsilon}}^{2} \\
& \leq C T k_{2}^{2} \int_{0}^{T} E \sup _{0 \leq s \leq t}\left\|u^{\epsilon}(s)-\mathbf{P} u(s)\right\|_{H^{\epsilon}}^{2} d t+4 T^{2} E \\
& \cdot \sup _{0 \leq t \leq T}\left\|f^{\epsilon}(s, \mathbf{P} u(s))-\mathbf{P} f(s, u(s))\right\|_{H^{\epsilon}}^{2}+2 E \\
& \cdot \sup _{0 \leq t \leq T}\left\|\int_{0}^{t}\left(e^{-A_{\epsilon}(t-s)} \mathbf{P}-\mathbf{P} e^{-A(t-s)}\right) f(s, u(s)) d s\right\|_{H^{\epsilon}} \cdot
\end{aligned}
$$

Here the contraction property of $e^{-A t}$, Hölder inequality, and conditions of (A.1) and (H.2) are used.

Denote $I_{21}=E \sup _{0 \leq t \leq T} \| \int_{0}^{t}\left(e^{-A_{\epsilon}(t-s)} \mathbf{P}-\mathbf{P} e^{-A(t-s)}\right) f(s$, $u(s)) d s \|_{H^{\epsilon}}^{2}$. Then we have

$$
\begin{aligned}
& I_{21}=\underset{0 \leq t \leq T}{E \sup _{t-r}} \| \int_{t}^{t}\left(e^{-A_{\epsilon}(t-s)} \mathbf{P}-\mathbf{P} e^{-A(t-s)}\right) \\
& \cdot f(s, u(s)) d s+\int_{0}^{t-r}\left(e^{-A_{\epsilon}(t-s)} \mathbf{P}-\mathbf{P} e^{-A(t-s)}\right) \\
& \quad \cdot f(s, u(s)) d s \|_{H^{e}}^{2} \leq 2 E \\
& \quad \cdot \sup _{0 \leq t \leq T} \| \int_{t-r}^{t}\left(e^{-A_{\epsilon}(t-s)} \mathbf{P}-\mathbf{P} e^{-A(t-s)}\right) \\
& \quad \cdot f(s, u(s)) d s \|_{H^{e}}^{2}+2 E
\end{aligned}
$$




$$
\begin{aligned}
& \cdot \sup _{0 \leq t \leq T} \| \int_{0}^{t-r}\left(e^{A_{\epsilon}(t-s)} \mathbf{P}-\mathbf{P} e^{-A(t-s)}\right) \\
& \cdot f(s, u(s)) d s \|_{H^{e}}^{2} \leq C(R, T) r^{2}+C(R, T) \\
& \cdot \frac{\tau(\epsilon)^{2}}{r^{2}}
\end{aligned}
$$

Hence we obtain

$$
\begin{aligned}
I_{2} \leq & 4 T^{2} k_{2} E \sup _{0 \leq t \leq T}\left\|u^{\epsilon}(s)-\mathbf{P} u(s)\right\|_{H^{\epsilon}}^{2}+4 T^{2} \tau_{1}(\epsilon) \\
& +C(R, T) r^{2}+C(R, T) \frac{\tau(\epsilon)^{2}}{r^{2}} .
\end{aligned}
$$

For $I_{3}$, use the maximal inequality (see [15]), contraction property of $e^{-A t},(\mathrm{~A} .1)$, and (H.2); then we have

$$
\begin{aligned}
I_{3} & =E \\
& \cdot \sup _{0 \leq t \leq T}\left\|\int_{0}^{t} e^{-A_{\epsilon}(t-s)} g^{\epsilon}\left(s, u^{\epsilon}(s)\right)-\mathbf{P} e^{-A(t-s)} g(s, u(s)) d w(s)\right\|_{H^{\epsilon}}^{2} \\
& \leq C E \\
& \cdot \sup _{0 \leq t \leq T}\left\|\int_{0}^{t} e^{-A_{\epsilon}(t-s)}\left(g^{\epsilon}\left(s, u^{\epsilon}(s)\right)-g^{\epsilon}(s, \mathbf{P} u(s))\right) d w(s)\right\|_{H^{\epsilon}}^{2} \\
& +C E \sup _{0 \leq t \leq T}\left\|\int_{0}^{t} e^{-A_{\epsilon}(t-s)}\left(g^{\epsilon}(s, \mathbf{P} u(s))-\mathbf{P} g(s, u(s))\right) d w(s)\right\|_{H^{\epsilon}}^{2} \\
& +C E \sup _{0 \leq t \leq T}\left\|\int_{0}^{t}\left(e^{-A_{\epsilon}(t-s)} \mathbf{P}-\mathbf{P} e^{-A(t-s)}\right) g(s, u(s)) d w(s)\right\|_{H^{\epsilon}}^{2} \\
& \leq C E \int_{0}^{T}\left\|e^{-A_{\epsilon}(t-s)}\left(g^{\epsilon}\left(s, u^{\epsilon}(s)\right)-g^{\epsilon}(s, \mathbf{P} u(s))\right)\right\|_{H^{\epsilon}}^{2} d s \\
& +C E \int_{0}^{T}\left\|e^{-A_{\epsilon}(t-s)}\left(g^{\epsilon}(s, \mathbf{P} u(s))-\mathbf{P} g(s, u(s))\right)\right\|_{H^{\epsilon}}^{2} d s \\
& +C E \int_{0}^{T}\left\|\left(e^{-A_{\epsilon}(t-s)} \mathbf{P}-\mathbf{P} e^{-A(t-s)}\right) g(s, u(s))\right\|_{H^{e}}^{2} d s \\
& \leq C k_{2}^{2} \int_{0}^{T} E \sup _{0 \leq s \leq t}\left\|u^{\epsilon}-\mathbf{P} u(s)\right\|_{H^{\epsilon}}^{2} d t+C T \tau_{1}(\epsilon)+C I_{31},
\end{aligned}
$$

where

$$
I_{31}=E \int_{0}^{T}\left\|\left(e^{-A_{\epsilon}(t-s)} \mathbf{P}-\mathbf{P} e^{-A(t-s)}\right) g(s, u(s))\right\|_{H^{\varepsilon}}^{2} d s .
$$

Let $l=t-s$. Notice that $t \geq s$ and $0 \leq t \leq T$. Then we have

$$
\begin{aligned}
I_{31}= & E \int_{0}^{t}\left\|\left(e^{-A_{\epsilon} l} \mathbf{P}-\mathbf{P} e^{-A l}\right) g(s, u(s))\right\|_{H^{e}}^{2} d l \\
= & E \int_{0}^{r}\left\|\left(e^{-A_{\epsilon} l} \mathbf{P}-\mathbf{P} e^{-A l}\right) g(s, u(s))\right\|_{H^{\varepsilon}}^{2} d l \\
& +E \int_{r}^{t}\left\|\left(e^{-A_{\epsilon} l} \mathbf{P}-\mathbf{P} e^{-A l}\right) g(s, u(s))\right\|_{H^{e}}^{2} d l \\
\leq & C(T, R) r+C(T, R) \frac{\tau(\epsilon)^{2}}{r^{2}} .
\end{aligned}
$$

By the above estimates of $I_{1}, I_{2}$, and $I_{3}$, we finally get

$$
\begin{aligned}
E \sup _{0 \leq t \leq T} & \left\|u^{\epsilon}(t)-\mathbf{P} u(t)\right\|_{H^{e}}^{2} \leq C(T) \\
\cdot & k_{2}^{2} \int_{0}^{T} E \sup _{0 \leq s \leq t}\left\|u^{\epsilon}(s)-\mathbf{P} u(s)\right\|_{H^{\epsilon}}^{2} d t+C(T, R) \\
& \cdot\left(r^{2}+r+\tau_{0}(\epsilon)+\tau_{1}(\epsilon)+\frac{\tau(\epsilon)^{2}}{r^{2}}+\frac{\tau(\epsilon)}{r}\right) .
\end{aligned}
$$

Use the Gronwall inequality we have the following estimate:

$$
\begin{gathered}
E \sup _{0 \leq t \leq T}\left\|u^{\epsilon}(t)-\mathbf{P} u(t)\right\|_{H^{\epsilon}}^{2} \leq e^{C(T) k^{2}}[C(T, R) \\
\left.\cdot\left(r^{2}+r+\tau_{0}(\epsilon)+\tau_{1}(\epsilon)+\frac{\tau(\epsilon)^{2}}{r^{2}}+\frac{\tau(\epsilon)}{r}\right)\right] .
\end{gathered}
$$

In particular,

$$
E \sup _{0 \leq t \leq T}\left\|u^{\epsilon}(t)-\mathbf{P} u(t)\right\|_{H^{\epsilon}}^{2} \longrightarrow 0,
$$

as $\epsilon \rightarrow 0$ and then $r \rightarrow 0$.

Remark 8. In this paper, we only consider the case in which Wiener process is scalar type; this result can not apply to the case of cylindrical Wiener processes. Note that if we concern the case of cylindrical Wiener processes, which relate to time and space, under perturbation of domain, cylindrical Wiener process is also perturbed which makes the situation more and more complicated.

\section{Competing Interests}

The authors declare that they have no competing interests.

\section{Acknowledgments}

The authors would like to thank Professor Jinqiao Duan for helpful discussions and comments. This work was supported by NSFs of China (nos. 11271013 and 11526196) and the Fundamental Research Funds for the Central Universities (HUST: 2014TS066).

\section{References}

[1] D. Henry, Perturbation of the Boundary in Boundary-Value Problems of Partial Differential Equations, vol. 318 of London Mathematical Society Lecture Note, Cambridge University Press, Cambridge, UK, 2005.

[2] D. Daners, "Dirichlet problems on varying domains," Journal of Differential Equations, vol. 188, no. 2, pp. 591-624, 2003.

[3] J. M. Arrieta and A. N. Carvalho, "Spectral convergence and nonlinear dynamics of reaction-diffusion equations under perturbations of the domain," Journal of Differential Equations, vol. 199, no. 1, pp. 143-178, 2004.

[4] W. Arendt, "Approximation of degenerate semigroups," Taiwanese Journal of Mathematics, vol. 5, no. 2, pp. 279-295, 2001. 
[5] F. Simondon, "Domain perturbation for parabolic quasilinear problems," Communications in Applied Analysis, vol. 4, no. 1, pp. $1-12,2000$.

[6] D. Daners, "Domain perturbation for linear and semi-linear boundary value problms," in Handbook of Differential Equations: Stationary Partial Differential Equations, vol. 6 of Handbook of Differential Equations, pp. 1-18, Elsevier, Amsterdam, Netherlands, 2008.

[7] N. Varchon, "Domain perturbation and invariant manifolds," Journal of Evolution Equations, vol. 12, no. 3, pp. 547-569, 2012.

[8] P. S. Ngiamsunthorn, "Invariant manifolds for parabolic equations under perturbation of the domain," Nonlinear Analysis: Theory, Methods \& Applications, vol. 80, pp. 28-48, 2013.

[9] M. Kunze and J. van Neerven, "Approximating the coefficients in semilinear stochastic partial differential equations," Journal of Evolution Equations, vol. 11, no. 3, pp. 577-604, 2011.

[10] D. Daners, "Perturbation of semi-linear evolution equations under weak assumptions at initial time," Journal of Differential Equations, vol. 210, no. 2, pp. 352-382, 2005.

[11] J. M. Arrieta and E. Santamaria, "Eetimates on the distance of inertial manifolds," Discrete and Continuous Dynamical Systems A, vol. 34, no. 10, pp. 3921-3944, 2014.

[12] G. D. Prato and J. Zabczyk, Stochastic Equation in Infinite Dimensions, Cambridge University Press, Cambridge, UK, 1922.

[13] P. L. Chow, Stochastic Partial Differential Equations, Chapman \& Hall/CRC, 2007.

[14] U. Mosco, "Convergence of convex sets and of solutions of variational inequalities," Advances in Mathematics, vol. 3, pp. 510-585, 1969.

[15] L. Gawarecki and V. Mandrekar, Stochastic Differential Equations in Infinite Dimensions, Springer, Berlin, Germany, 2011. 


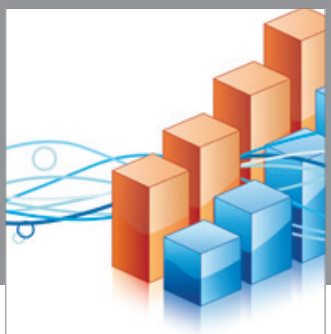

Advances in

Operations Research

vatem alat4

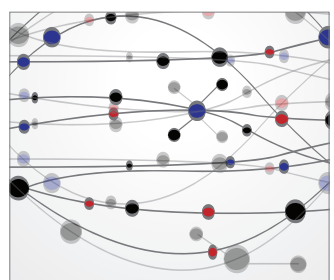

\section{The Scientific} World Journal
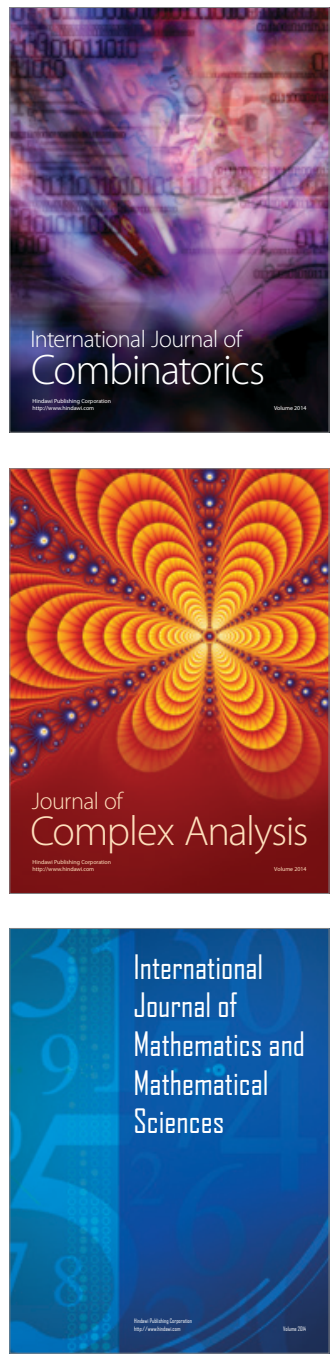
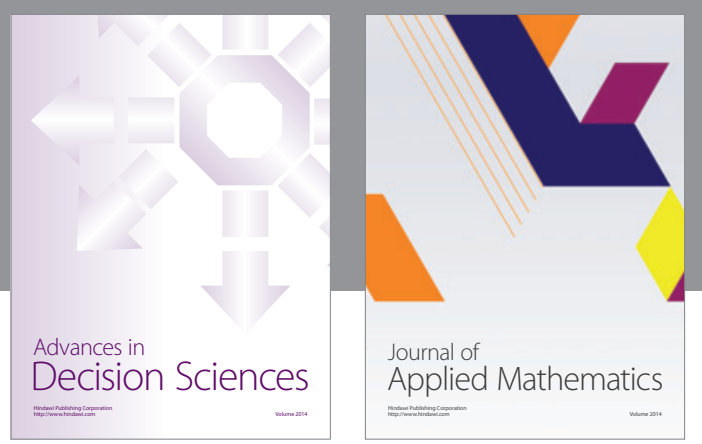

Algebra

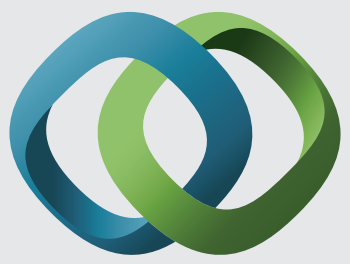

\section{Hindawi}

Submit your manuscripts at

http://www.hindawi.com
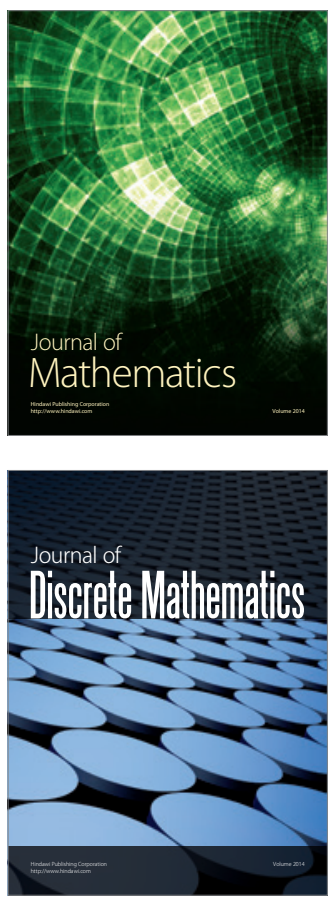

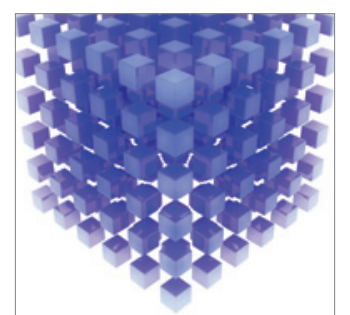

Mathematical Problems in Engineering
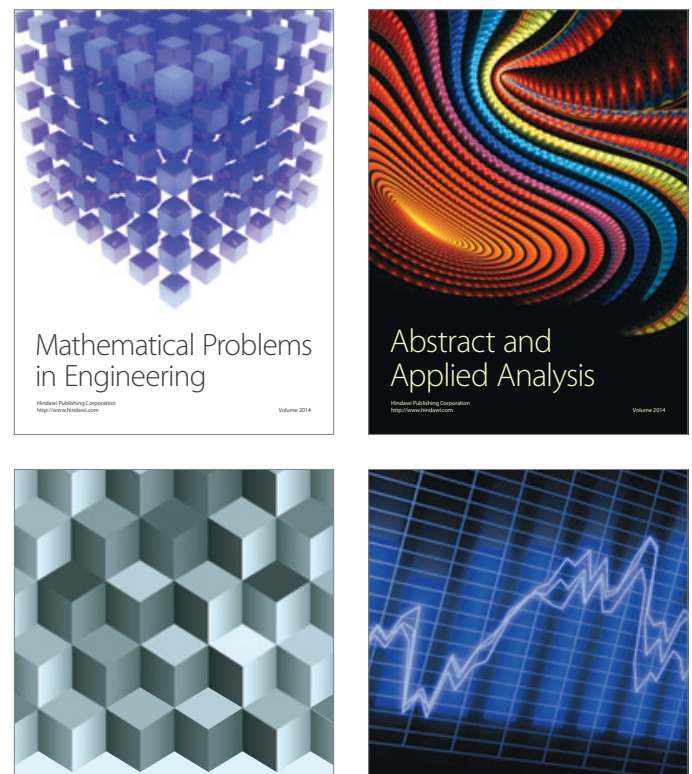

Journal of

Function Spaces

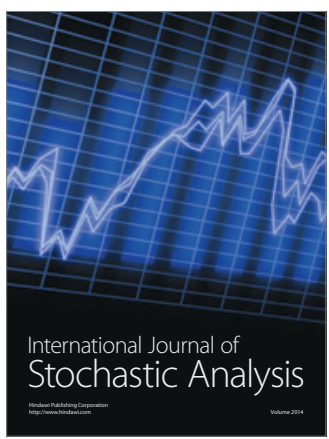

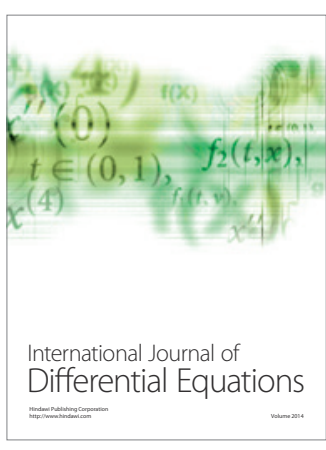
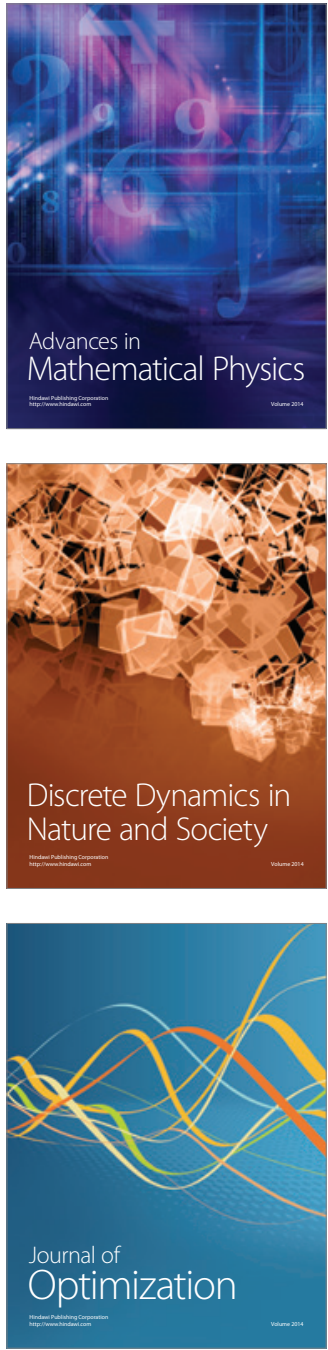